\title{
Crystallization of Poly(E-caprolactone)-block-Polystyrene Copolymers from Glassy Microdomain Structures
}

\author{
Shuichi Nojima, ${ }^{\dagger}$ Hiroshi Kakihira, Satoshi Tanimoto, \\ Hisayuki Nakatani, ${ }^{*}$ and Shintaro SaSakI \\ School of Materials Science, Japan Advanced Institute of Science \\ and Technology (JAIST), Tatsunokuchi, Ishikawa 923-1292, Japan \\ * Center for New Materials, Japan Advanced Institure of Science and Technology (JAIST), \\ Tatsunokuchi, Ishikawa 923-1292, Japan
}

(Received May 10, 1999)

\begin{abstract}
KEY WORDS Crystalline-Amorphous Diblock Copolymer / Microdomain Structure / Glass Transition / Crystallization /
\end{abstract}

Crystallization of block copolymers is significantly affected by the microdomain structure existing in the melt. ${ }^{1}$ When the glass transition temperature $T_{\mathrm{g}}$ of the amorphous block is higher than the melting temperature $T_{\mathrm{m}}$ of the crystallizable block, the microdomain structure freezes substantially when the constituent block crystallizes. As a result, the crystallization is restricted to occur within the microdomain structure. ${ }^{2-9}$ In the morphological studies of such systems, it is of great importance to get information about the microdomain structure. Small-angle X-ray scattering (SAXS) measurements are generally used to characterize the microdomain structure formed in block copolymers consisting of amorphous chains, ${ }^{10}$ but this method is not available when the electron density difference between domains is extremely small. This is the case for some crystalline-amorphous diblock copolymers investigated so far, such as poly $(\varepsilon-$ caprolactone)-block-polystyrene (PCL- $b$-PS), ${ }^{2,3,8,9}$ poly(tetrahydrofuran)-block-PS (PTHF-b-PS), ${ }^{11}$ and poly(ethylene oxide)-block-PS (PEO-b-PS). ${ }^{12}$ Therefore, there is no study to relate the microdomain structure with the final morphology for the block copolymers with $T_{\mathrm{g}}>T_{\mathrm{m}}$.

We have recently reported the effects of $T_{\mathrm{g}}$ and copolymer composition on the crystallization of PCL$b$-PS copolymers. ${ }^{8,9}$ When the volume fraction of PCL blocks, $\phi_{\text {PCL }}$, is less than 0.26 , the PCL block does not crystallize at all, while the crystallinity increases abruptly between $0.34 \leq \phi_{\mathrm{PCL}} \leq 0.60$ toward a constant value. $^{2,8}$ This significant change in the crystallinity should relate intimately with the microdomain structure; we can suppose intuitively that when the PCL block is a matrix it crystallizes easily, whereas when it is an isolated phase surrounded by the amorphous PS matrix the crystallization will be extremely disturbed because of the spatial restriction.

In this study we try to characterize the microdomain structure of PCL- $b$-PS in the melt by SAXS. For this purpose, we add a small amount of poly(vinyl chloride) (PVC) to the PCL block ${ }^{13}$ to increase the electron density of the PCL domain. The repeating distance of the microdomain structure, which is straightforwardly obtained from SAXS measurements, is evaluated and compared with that of the morphology after the crystallization of PCL blocks. From these results, we elucidate the effect of existing microdomain structures on the final morphology formed at various temperatures in crystalline-amorphous diblock copolymers with a high $T_{\mathrm{g}}$ amorphous block.

\section{EXPERIMENTAL}

\section{Materials and Sample Preparation}

PCL- $b$-PS copolymers used in this study were synthesized by a successive anionic polymerization under vacuum. Details of the synthesis and molecular characterization are described elsewhere. 8,9 Two PCL$b$-PS copolymers, designated by CL64 and CL70, are used in which the PCL block is a major component because the PCL block crystallizes sufficiently only at a limited composition range $\left(\phi_{\mathrm{PCL}} \geq 0.6\right) .{ }^{9}$ The results of molecular characterization are shown in Table I. CL64 has a lamellar microdomain structure in the melt judging from $\phi_{\mathrm{PCl}}$ and the TEM micrograph after the crystallization of PCL blocks and CL70 has a cylindrical microdomain structure (see Figure 3).

The crystallization of PCL blocks in CL64 and CL70 was performed by quenching the sample from the temperature above $T_{\mathrm{m}}$ into various crystallization temperatures $T_{\mathrm{c}}$ ranging from 20 to $50^{\circ} \mathrm{C}$. The crystallization of CL64 was also performed by the solution-casting method to compare the final morphology with that of the quenched sample, where toluene, good for both the blocks, was used for the casting solvent and evaporated gradually at various $T_{\mathrm{c}}$ to prepare a crystallized thin film of PCL- $b$-PS.

\section{SAXS Measurements}

SAXS measurements were performed with a pointfocusing optics and a one-dimensional position sensitive proportional counter. Details of the optics, instrumentation, and data processing are described elsewhere. ${ }^{14,15}$ The Lorentz-corrected SAXS curves were finally obtained as a function of wave number $s$ defined by

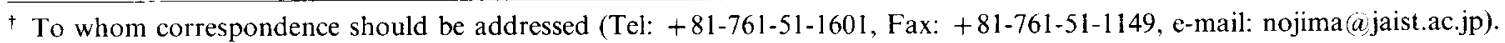


Table I. Characterization of polymers used in this study

\begin{tabular}{cccccc}
$\begin{array}{c}\text { Sample } \\
\text { code }\end{array}$ & Polymer & Source & Total $M_{n}{ }^{\mathrm{c}}$ & $M_{w} / M_{n}{ }^{\mathrm{c}}$ & PCL $:$ PS \\
\cline { 2 - 5 } CL64 & PCL- $b$-PS & a & $30000^{\mathrm{d}}$ & 1.29 & $64: 36$ \\
CL70 & PCL- $b$-PS & $\mathrm{a}$ & 39000 & 1.35 & $70: 30$ \\
PVC1 & PVC & b & 40000 & 1.95 & -
\end{tabular}

a Synthesized in our laboratory. ${ }^{\text {b }}$ Obtained from Scientific Polymer Products, Inc. " Determined by GPC with PS as a standard. ${ }^{\mathrm{d}}$ Determined by osmometric pressure. ${ }^{c}$ Determined by ${ }^{1} \mathrm{H}$ NMR.

$s=(2 / \lambda) \sin \theta$, where $\lambda$ is the wave length of the X-ray $(\lambda=0.154 \mathrm{~nm})$ and $2 \theta$ is the scattering angle. In this study, we focussed our attention only on the repeating distance of the morphology.

The electron density difference between the amorphous PCL $\left(337 \mathrm{enm}^{-3}\right.$ at $\left.100^{\circ} \mathrm{C}^{16}\right)$ and amorphous PS $\left(332 \mathrm{e} \mathrm{nm}^{-3}\right.$ at $100^{\circ} \mathrm{C}^{17}$ ) is unfortunately extremely small, so we cannot expect the distinct scattering from the microdomain structure. To make the scattering conspicuous for getting information about the microdomain structure, we added a small amount of PVC (designated by $\mathrm{PVCl}$ in Table I) into the sample, which resided selectively in the PCL domain to enhance the electron density of the PCL domain. The repeating distance (or domain spacing) of the microdomain structure $D$ thus evaluated by SAXS was extrapolated to $\phi_{\mathrm{PVC}}=0$ to get the domain spacing of pure PCL- $b$-PS $D_{0}$, where $\phi_{\mathrm{PVC}}$ represents the volume fraction of $\mathrm{PVC}$ in the sample. Each PCL- $b$-PS/PVC sample was cast from tetrahydrofuran solution at the temperature above $T_{\mathrm{m}}$. The morphology after the crystallization of PCL blocks, on the other hand, could be observed satisfactorily by SAXS since there is an enough electron density difference between PCL crystals $\left(393 \mathrm{e} \mathrm{nm}^{-318}\right)$ and amorphous PS blocks $\left(344 \mathrm{e} \mathrm{nm}^{-3}\right.$ at $\left.40^{\circ} \mathrm{C}^{17}\right)$.

Transmission Electron Microscopy (TEM) Observations The morphology of CL64 and CL70 crystallized at room temperature $\left(<T_{\mathrm{m}}\right)$ was observed by TEM. The sample was first trimmed at $-140^{\circ} \mathrm{C}$ with a glass knife, stained with $\mathrm{RuO}_{4}$ vapor for $36 \mathrm{~h}$ at room temperature, and finally microtomed with a diamond knife into a thin film with a thickness of $c a .90 \mathrm{~nm}$. The amorphous region (PS block and amorphous PCL block) was preferentially stained by $\mathrm{RuO}_{4} \cdot{ }^{19}$ TEM images were obtained with a Hitachi $\mathrm{H}-7100$ TEM operating at $100 \mathrm{kV}$. The repeating distance of the morphology was roughly estimated from the TEM image.

\section{DSC Measurements}

A Perkin Elmer Pyris 1 DSC was used to confirm the crystallization of PCL blocks in PCL- $b$-PS. The sample crystallized at various $T_{\mathrm{c}}$ for $24 \mathrm{~h}$ was heated with a heating rate of $5^{\circ} \mathrm{C} \mathrm{min}{ }^{-1}$ and the endothermic peak due to the melting of the PCL block was observed. The melting temperature $T_{\mathrm{m}}$ and crystallinity were also evaluated, ${ }^{9}$ but these values were not used in this study.

\section{RESULTS AND DISCUSSION}

\section{Microdomain Structure Existing in the Melt}

The original SAXS curve (before any corrections) for

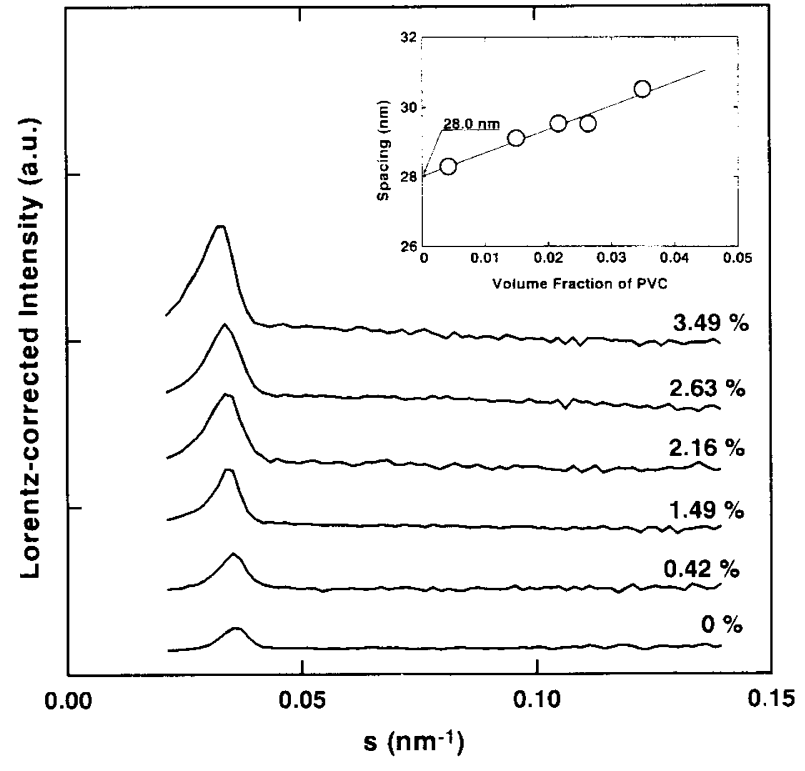

Figure 1. Lorentz-corrected SAXS intensity plotted against $s$ $(=(2 / \lambda) \sin \theta, 2 \theta$ is the scattering angle and $\lambda$ is the $\mathrm{X}$-ray wavelength $(=0.154 \mathrm{~nm})$ ) for CL70/PVC1 blends with various $\mathrm{PVC}$ vol\% indicated on each curve. The measurement was performed at $c a .64^{\circ} \mathrm{C}$, which is higher than $T_{\mathrm{m}}$ of the PCL block. The inset shows the plot of $D$ against $\phi_{\mathrm{PVC}}$ to evaluate $D_{0}$ for CL70.

the molten CL64 and CL70 had a faint intensity peak from the microdomain structure on the background scattering, and we expected much error when evaluating the peak position after various corrections. In particular, the subtraction of a large background scattering would affect significantly the peak position of the remaining SAXS curve. The peak became clear with increasing $\phi_{\text {PVC }}$ and it was reliable to evaluate the peak position. Figure 1 shows the Lorentz-corrected SAXS curves obtained from the molten CL70/PVC1 blends with various $\phi_{\mathbf{P V C}}$. The intensity peak grows larger and broader and the position shifts to the lower angle with increasing $\phi_{\mathbf{P v C}}$, which is attributed to the added PVC in the system. It is important here that the added PVC does not change the type of the microdomain structure, for which we restricted $\phi_{\mathrm{PVC}}$ less than 0.04 to make the microdomain structure unchanged by adding PVC.

The inset of Figure 1 shows the plot of $D$ evaluated from the peak position against $\phi_{\mathbf{P V C}}$. The plot makes a straight line and it is easy to extrapolate this line to $\phi_{\mathrm{PVC}}=0 .{ }^{20}$ The value of $D_{0}$ thus evaluated is $25.2 \pm 0.2 \mathrm{~nm}$ for CL64 and $28.0 \pm 0.3 \mathrm{~nm}$ for CL70. It was, however, impossible to decide the type of the microdomain structure only from the SAXS curve because higher intensity peaks were absent owing to the irregularity of the PVC-added microdomain structure. Figure 1 gives the alternative method to evaluate the angular position of the SAXS peak even for weakly scattering systems.

\section{Repeating Distances Before and After Crystallization of PCL Blocks}

We observed the endothermic peak in the DSC curve of CL64 and CL 70 crystallized at $T_{\mathrm{c}} \leq 50^{\circ} \mathrm{C}$ for $24 \mathrm{~h}$. This result, along with the definite $\mathrm{SAXS}$ intensity peak arising from the electron density difference between PCL crystals and amorphous PS and PCL regions, indicates the 


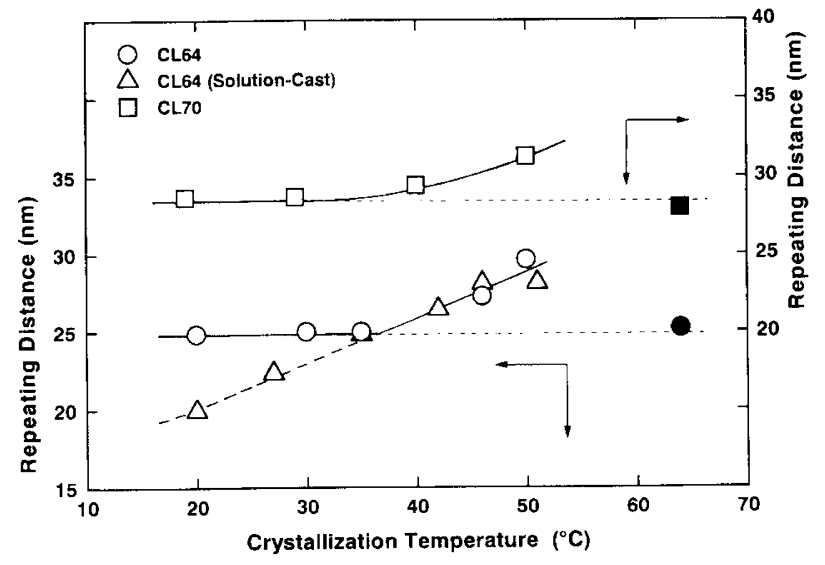

Figure 2. The repeating distance plotted against $T_{\mathrm{c}}$ for quenched CL64 $(O)$, solution-cast CL64 $(\triangle)$, and quenched CL70 ( $\square)$. The closed symbols represent the values of $D_{0}$ for CL64 and CL70.

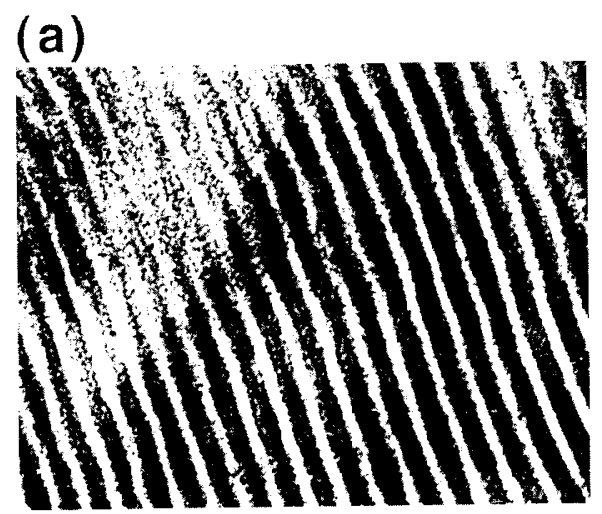

(b)

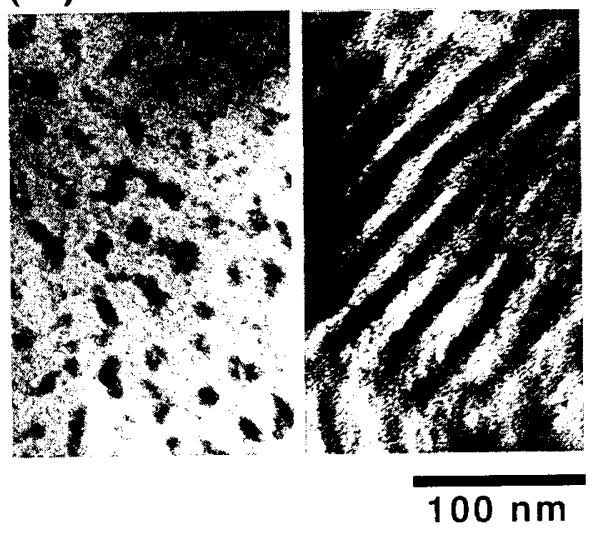

Figure 3. TEM micrograph showing (a) the lamellar morphology of CL64 and (b) hexagonally packed cylinder of CL70. The white region is PCL crystals unstained by $\mathrm{RuO}_{4}$.

crystallization of PCL blocks in the system. The repeating distance of the crystallized PCL- $b$-PS, $L$, was easily evaluated from the peak position. Figure 2 shows the $T_{\mathrm{c}}$ dependence of $L$ for CL64 and CL70, together with $D_{0}$ obtained by the method described above. The values of $L$ at $T_{\mathrm{c}} \leq 35^{\circ} \mathrm{C}$ for CL64 and CL70 are constant and identical with each $D_{0}$, suggesting that the PCL block crystallizes within the microdomain structure existing in the melt. The morphology formed at room temperature $\left(<35^{\circ} \mathrm{C}\right)$ was observed by TEM for CL64 and CL70, and found the lamellar morphology for CL64 and cylindrical morphology for CL70 (Figure 3), as expected.
The repeating distance evaluated from Figure 3 is $c a$. $20 \mathrm{~nm}$ for CL64 and ca. $30 \mathrm{~nm}$ for CL70, which are in good agreement with $D_{0}$ (closed symbols in Figure 2). Figure 3 , together with $L$ observed at $T_{\mathrm{c}} \leq 35^{\circ} \mathrm{C}$, confirms that the PCL block crystallizes within the microdomain structure without any disruption of this structure.

When $T_{\mathrm{c}} \geq 35^{\circ} \mathrm{C}, L$ increases gradually with increasing $T_{\mathrm{c}}$ both for CL64 and CL70. The $T_{\mathrm{c}}$ dependence of $L$ for CL64 at $T_{\mathrm{c}} \geq 35^{\circ} \mathrm{C}$ is similar with that for the solution-cast CL64. The morphology formed by the solution-casting method is believed to be more stable and less affected by the pre-existing microdomain structure. That is, when $T_{\mathrm{c}} \geq 35^{\circ} \mathrm{C}$, the crystallization of the PCL block in the quenched CL64 makes the morphology more stable by disrupting or distorting the microdomain structure. A similar $T_{\mathrm{c}}$ dependence of the final morphology was reported by Register et al. ${ }^{21}$ for a weakly segregated polyethylene-block-poly(3-methyl-1butene), where the kinetic factor during crystallization controlled the final morphology; fast cooling to lower $T_{\mathrm{c}}$ resulted in the crystallization confined in the microdomain structure while slow cooling disrupted the microdomain structure to yield the lamellar morphology favorable to crystalline-amorphous diblocks. The morphology formation in our samples, however, would relate intimately to the glass transition of the PS domain and the competitive effects between the glass transition and crystallization may play a crucial role in determining the final morphology. However, we could not evaluate $T_{\mathrm{g}}$ of the PS domain successfully by DSC measurements.

In summary, the final morphology formed in PCL- $b$-PS by the crystallization of PCL blocks is dependent on $T_{\mathrm{c}}$; when $T_{\mathrm{c}}$ is lower than $35^{\circ} \mathrm{C}$ the microdomain structure does not change and the PCL block crystallizes within it, whereas when $T_{\mathrm{c}}$ is higher than $35^{\circ} \mathrm{C}$ the crystallization leads to the disruption or distortion of the existing microdomain structure. The $T_{\mathrm{g}}$ of the amorphous domain would affect significantly the difference in this morphology formation. Thus, the morphology formation of crystalline-amorphous diblock copolymers is extremely complicated, which is dependent on many factors such as the molecular weight (segregation strength between different blocks), microdomain structure, and glass transition of the amorphous block. Therefore, it is necessary to elucidate these effects on the crystallization behavior and final morphology to understand the crystallization mechanism of crystalline-amorphous diblock copolymers.

Acknowledgments. This work was supported in part by Tokuyama Science Foundation and by Grants-in-Aid for Scientific Research (No. 09650992) from the Ministry of Education, Science, Sports and Culture of Japan.

\section{REFERENCES AND NOTES}

1. I. W. Hamley, "The Physics of Block Copolymers," Oxford University Press, Oxford 1998.

2. J. J. Herman, R. Jerome, P. Teyssie, M. Gervais, and B. Gallot, Makromol. Chem., 182, 997 (1981).

3. J. Heuschen, R. Jerome, and P. Teyssie, J. Polym. Sci., B27, 523 (1989).

4. L. Liu, H. Li, B. Jiang, and E. Zhou, Polymer, 35, 5511 (1994).

5. L. Liu, B. Jiang, and E. Zhou, Polymer, 37, 3937 (1996). 
6. V. Balsamo, F. Gyldenfeldt, and R. Stadler, Macromol. Chem. Phys., 197, 3317 (1996).

7. L. Liu, F. Yeh, and B. Chu, Macromolecules, 29, 5336 (1996).

8. S. Nojima, H. Tanaka, A. Rohadi, and S. Sasaki, Polymer, 39, 1727 (1998).

9. S. Nojima, M. Fujimoto, H. Kakihira, and S. Sasaki, Polym. J., 30, 968 (1998).

10. F. S. Bates and G. H. Fredrickson, Annu. Rev. Phys. Chem., 41, 525 (1990).

11. T. Shiomi, H. Tsukada, H. Yoshise, K. Takenaka, and Y. Tezuka, Polym. Prepr., Jpn., 46, 808 (1997).

12. M. Gervais and B. Gallot, Polymer, 22, 1129 (1981).

13. F. B. Khambatta, F. Warner, T. Russell, and R. S. Stein, J. Polym. Sci., Polym. Phys. Ed., 14, 1391 (1976).

14. S. Nojima, K. Hashizume, A. Rohadi, and S. Sasaki, Polymer, 38, 2711 (1997).
15. S. Nojima, M. Kuroda, and S. Sasaki, Polym. J., 29, 642 (1997).

16. V. Crescenzi, G. Manzini, G. Galzolari, and C. Borri, Eur. Polym. $J ., 8,449$ (1972).

17. M. J. Richardson and N. G. Savil, Polymer, 18, 3 (1977).

18. K. Ito and Y. Yamashita, Macromolecules, 11, 68 (1978).

19. A. K. Khandpur, C. W. Macosko, and F. S. Bates, J. Polym. Sci., B33, 247 (1995).

20. Precisely, the plot of $D v s . \phi_{\mathbf{P v c}}{ }^{1 / 2}$ is necessary to obtain $D_{0}$ for CL $70^{22}$ because the microdomain structure is a hexagonally packed cylinder. However, we used the $D v s . \phi_{\mathrm{PvC}}$ plot to evaluate $D_{0}$ because of small $\phi_{\mathrm{PVC}}$.

21. D. J. Quiram, R. A. Register, and G. R. Marchand, Macromolecules, 30, 4551 (1997).

22. H. Tanaka, H. Hasegawa, and T. Hashimoto, Macromolecules, 24, 240 (1991). 